\title{
Alles muss raus: Die politische Logik des „Potenzials“
}

\author{
STEPHAN LESSENICH
}

$D$ er gesellschaftspolitische Diskurs im flexiblen Kapitalismus der Gegenwart hat eine neue semantische Lieblingsfigur: das Potenzial. Wo man auch hinschaut, wer auch immer sich äußert - überall und von allen Seiten werden Potenziale ausgemacht, die es zu entdecken und zu entwickeln, zu heben und zu nutzen gilt. Wo fing das an und wann?

Vermutlich mit dem weiblichen Humankapital, das zumal in der Bundesrepublik lange Zeit zwecks Kinder-, Eltern- und Ernährerehemannbetreuung in Privathaushalten brachlag und nun schon seit geraumer Zeit im Zuge einer eigentümlichen, feministisch-produktivistischen Doppelbewegung auf Arbeitsmärkte mobilisiert wird. Eine ähnlich ambivalente, liberal-progressive Koalition hat sich die soziale Integration - und das heißt heute auf gut deutsch stets: Arbeitsmarktinklusion - migrantischer Milieus auf die Fahnen geschrieben, weil die Wettbewerbsbedingungen der globalisierten Wissensgesellschaft es erzwingen, alle potenziell fähigen Köpfe bestmöglich auszubilden und volkswirtschaftlich gewinnbringend einzusetzen. Zudem herrscht mittlerweile große wissenschaftlich-politische Einigkeit darüber, dass alte Menschen heutzutage über ein Portfolio mannigfaltiger Ressourcen verfügen, die produktiv zu nutzen angesichts des demografischen Wandels und der Arbeitsangebotsnöte einer alternden Gesellschaft das Gebot der Zukunft ist - was selbstverständlich umso mehr für die ausgedünnte junge Generation gilt, die von Kindesbeinen an zum Hoffnungsträger einer Reproduktion des historisch gewachsenen wirtschaftlichen Wachstumsmodells erkoren und mit entsprechenden öffentlichen wie privaten Potenzialentdeckungs- und-förderungsinterventionen überzogen wird. Für sie alle wie für jede Einzelne und jeden Einzelnen in jeder Lebensphase gilt es, etwas aus ihnen bzw. sich selbst zu machen, sprich: sie bzw. sich in ein produktives, nützliches, marktfähiges Mitglied der Gesellschaft zu verwandeln.

Wo in dieser Gesellschaft also „Potenziale" sind oder vermutet werden, da heißt die Standarddevise: Ran an den Speck! Das gilt - ganz wörtlich - für gesundheitspolitische Fitness-, Bewegungs- und Ernährungsprogram me (können wir nicht alle ein wenig mehr "für uns" tun?) wie auch - im übertragenen Sinne - für die „Verschlankung“ von Betrieben, Verwaltungen und Organisationen (wo es grundsätzlich immer noch Möglichkeiten weiterer „Effizienz"-Steigerung gibt). Und es gilt natürlich für all jene unendlich zahlreichen und vielfältigen Fälle, in denen Menschen irgendwie noch mehr aus sich herausholen könnten: sich häufiger bewerben, sich weiter qualifizieren, lebenslang lernen und das Leben noch stärker der Arbeit verfügbar machen. Und wenn all dies als „natürlich“ gilt, dann auch deshalb, weil die Analogie zur menschlichen Naturbeherrschung auf der Hand liegt: Die menschlichen Potenziale sind wie Bodenschätze, die man nicht unter der Erde lassen darf, sondern ans Tageslicht bringen muss, und dies zwar („leider") nicht rückstandsfrei, aber doch möglichst restlos - alles muss raus! Wem heute Potenziale attestiert werden, der sollte sich also warm anziehen. Denn wenn die politische Potenzialbeschwörungsmaschinerie anspringt, dann haben die Bürgerinnen und Bürger mit einem weiteren Schub der Ökonomisierung ihrer Lebensverhältnisse zu rechnen. Und wie bei der Ausbeutung der natürlichen Reichtümer keine Kosten und Mühen gescheut werden, um auch noch die tiefsten Erdschichten zu durchdringen und die planetarischen Potenziale wirklich bis zum Letzten auszuschöpfen, so werden auch immer neue und komplexere sozial-politische Interventionsformen erdacht, um die bislang verborgenen oder vorenthaltenen persönlichen Potenziale der Leute einer wirtschaftlichen bzw. gesellschaftlichen Verwertung zuzuführen: "fracking" ist auch eine Sozialtechnologie.

In der bisweilen geradezu wahnhafte Züge annehmenden kollektivindividuellen Suche nach dem produktiven Potenzial spielen alternative Wertbestimmungen des Sozialen - Fragen der individuellen Selbstbestimmung und der kollektiven Solidarität, der Befreiung aus ökonomischer Abhängigkeit und sozialer Unmündigkeit - keine politische Rolle mehr. Oder mehr (bzw. weniger) noch: Sie werden unter Rückgriff auf eine soziologische Zauberformel, mit der beliebten Rede von sozialer "Inklusion“, der produktivistischen Potenzialnutzungsstrategie gleichgeschaltet. Denn wer integraler Teil des ökonomischen Marktgeschehens, vulgo gesellschaftlich „inkludiert“ ist, kommt dadurch quasi-automatisch - so das Credo der herrschenden (Sozial-)Politik - in den Genuss von Teilhabe und Anerkennung, Autonomie und Partizipation. Nur genutzte Potenziale sind gute Potenziale - die Nutzungsbedingungen allerdings sind durch die Geschäftsordnung der Marktgesellschaft vorgegeben.

Alles muss raus: Politik im flexiblen Kapitalismus betreibt einen ständigen Winterschlussverkauf der Potenziale. Ob es in dieser Welt aber jemals wieder Sommer werden wird?

STEPHAN LESSENICH, Dr. habil., ist Professor für Vergleichende Gesellschaftsund Kulturanalyse und Ko-Direktor der DFG-Kollegforschergruppe "Postwachstumsgesellschaften" am Institut für Soziologie der Friedrich-SchillerUniversität Jena.

stephan.lessenich@uni-jena.de 